\title{
Taking into Account Outer Hydrodynamic Pressure in Differential Equation of Variable Flow Rate Fluid Flow for Distributive Pressure Pipelines
}

\author{
Volodymyr V. Cherniuk \\ Lviv Polytechnic National University \\ Institute of Civil and Environmental Engineering, Department of Hydraulics and Sanitary Engineering \\ e-mail: chernyuk@1p.edu.ua
}

\begin{abstract}
In the differential equation of variable flow rate fluid flow a component which takes into account outer hydrodynamic pressure is introduced. The variables of the equation are expressed in terms of full operating head and in terms of independent distance along the axis of the stream, i. e. this equation is reduced to a singlevariable equation.
\end{abstract}

Key words: Pressure distributive pipeline, variable flow rate fluid flow.

\section{Introduction}

Pressure distributive pipelines (DP) with discrete dispensation of fluid along the path are widely used in irrigation (systems of drip, subsurface, and sprinkling irrigation , furrow watering), ventilation (tide systems), water supply and water draining (distributive pipe systems of purification works, dispersed discharges of treated waste water), water transport (distributive filling systems of navigation locks and high clearance dry docks), metallurgical industry (cooling systems), in apparatus of oil-processing, oil-chemical, chemical, and food industries and in other branches of engineering. There are different methods of calculation for pressure distributive pipelines. However, their exact hydraulic calculation can be made only by means of the theory of variable mass hydraulics [1, page 4].

The creator of the theory of variable mass body motion is I. V. Mescherskiy. He stated the problem, deduced main equations, and showed branches of their application. The prof. I. V. Mescherskiy's works "Dynamics of a point of variable mass" (variable mass point) (1987) and "Equation of motion of a variable mass point in general case" (1904) have laid foundation of a new branch of theoretical mechanics. Basing on this branch, rocket engineering and hydraulics of variable mass fluid have started their successful development. 
On the basis of the equations obtained by prof. I. V. Mescherskiy for a variable mass point, prof. I. V. Makaveev in 1928 for the first time deduced a general equation of variable flow rate fluid flow (DEVFRFF), and he applied it for solving the problem of hydraulic jump. In 1937, prof. Ya. T. Nen'ko obtained DEVFRFF for the whole stream, and he applied it for solving problems of calculation of perforated DP with continuous fluid dispensation along, the path. He also established the criteria of DP classification with respect to their length. The same year, prof. I. M. Konovalov obtained the DEVFRFF on the basis of the law of conservation of momentum, and he applied it for solving problems of fluid flow in pipelines and canals. Variable flow rate fluid flow is also studied by other investigators [2, page 3-4].

\section{Analysis of DEVERFF}

According to prof. G. A. Petrov's statements, the general DEVERFF which describes simultaneous collection and dispensation of fluid by a pipeline with holes in its walls is of the form [2, page 17]:

$$
\frac{\alpha_{o}}{g} V d V+d\left(\frac{p}{\gamma}\right)+d z+i_{f} d x+\frac{V^{2}}{g} d \alpha_{o}+\frac{\alpha_{o}\left(V-v_{1} \cos \beta\right) V}{g} \cdot \frac{d Q_{1}}{Q}+\frac{\alpha_{o}\left(v_{2} \cos \varphi-V\right) V}{g} \cdot \frac{d Q_{2}}{Q}=0,
$$

where the sixth term describes the adding of mass, and the seventh term describes its loss; $Q_{1}$ is the flow rate of the fluid which is being added to the stream in the pipe; $Q_{2}$ is that which is leaving the stream in the pipe; $d z=\sin \psi \cdot d x$ is the geometric elevation of the axis of perforated pipeline in its interval of $d x$ length; $\psi$ - is the angle of the inclination of the pipeline axis to horizontal; $i_{f} d x=d h_{x}$ is the head loss for friction along the pipeline; $\beta$ and $\varphi$ are angles between the vector $\vec{V}$ of the velocity of the main stream inside the perforated pipeline and vectors $\vec{v}$ of jets which flow in and out respectively.

Prof. G. A. Petrov holds that it will be no considerable error if we take $\alpha_{o}$ as a constant [2, page 17]. Then the fifth term $\frac{V^{2}}{g} d \alpha_{o}$ in Equation (1) becomes zero. Thus, in the case of only loss of fluid, i.e. for DP, the equation (1) takes the form:

$$
d\left(\frac{\alpha_{o} V^{2}}{2 g}\right)+\frac{d p}{\rho g}+d z+i_{f} d x+\frac{\alpha_{o}(v \cos \varphi-V) \cdot V}{g} \frac{d Q}{Q}=0 .
$$

For cylindrical DP, when $\omega=$ const, $Q=\omega V, d Q=\omega d V$, Equation (2) is simplified to the following form:

$$
\frac{\alpha_{o}(v \cos \varphi-2 V) \cdot d V}{g}+\frac{d p}{\rho g}+\sin \psi \cdot d x+d h_{x}=0
$$

In practice, there occurs operation of DP which is situated in a stream of fluid which washes it from outside. According to such scheme, a mixer-pipeline consisting of two coaxial pipes operates: the inner pipe of it is filled with one fluid, the outer pipe with another fluid. Through the holes in the wall, the fluid from the inner pipe flows into the space between the pipes 
where it mixes with the other fluid [3]. According to the principle of submerged DP, dispersed discharge of treated waste water into a river is conducted (Fig. 1) [4, 5].

However in Equations (1)-(3) the pressure of outer fluid stream is not taken into account. Incomplete taking into account constructional parameters of a distributive pipeline and that of hydrodynamic peculiarities of variable flow rate stream leads to considerable miscalculations during designing DP; such miscalculations cause reduction in effectiveness of its operation [6, page 3].

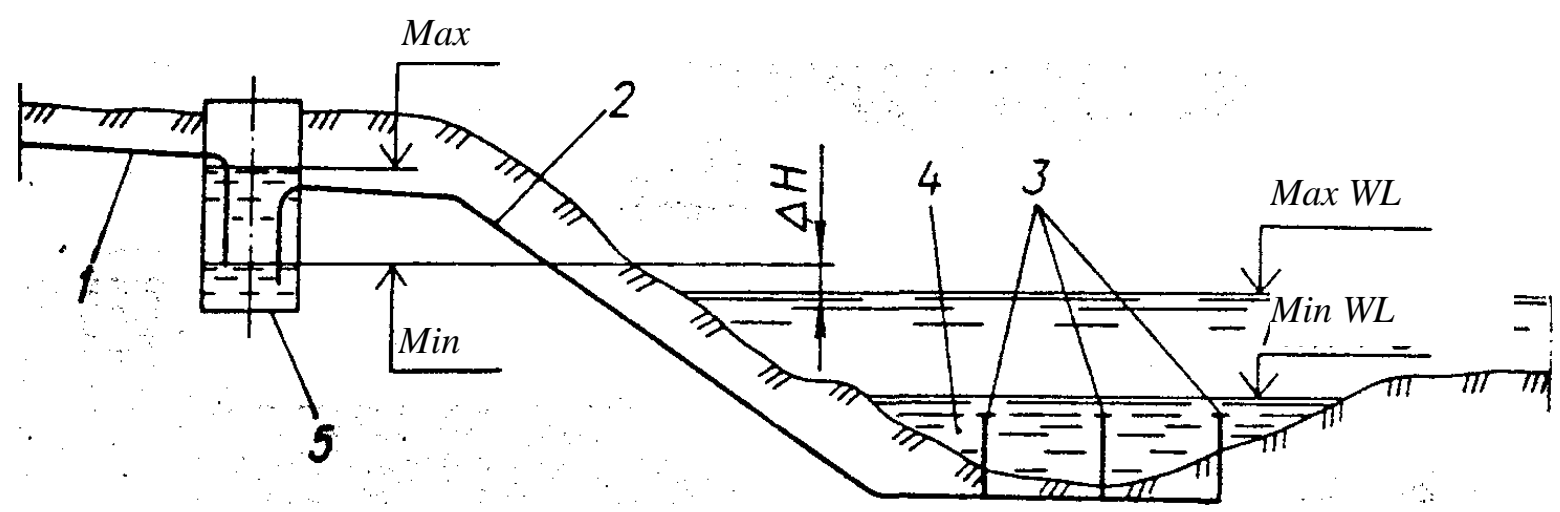

Figure1: Water discharge plant with distributive pipeline in water stream: 1,2 - pipelines; 3 water outlets, 4 - river, 5 - adjusting well

The aim of this work is to introduce a term describing hydrodynamic pressure of outer fluid stream into DEVFRFF in order to improve the exactness of calculation of pressure DP.

\section{Generalization of DEVFRFF}

Professor G. A. Petrov holds that, in the places of branching, the fluid flow is a rather complicated phenomenon, and it is impossible to take into account all its elements [2, page 55]. Moreover, to solve this problem by means of exact methods of hydromechanics is impossible yet [2, pages 44-45]. Under to-day's state of art in hydraulics, to previously determine the law of change of the coefficient $\alpha_{0}$ is also impossible [2, page 17]. Besides, a correction of $\alpha_{0}^{\prime}$ which takes into account the non-uniformity of distribution of velocities in a free cross-sectional area of an outflowing jet can, in general case, differ from the correction of $\alpha_{0}$ for the main stream. Moreover, in the interval of branching, there acts the wall reaction $R_{w}$, which is opposite to the outlet hole. The reaction $R_{w}$ is perpendicular to this wall, and its projection on the main stream axis can be considered to equal zero [2, page 47]. G. A. Petrov also introduces a force $\mathrm{S}$ which is to be substituted for the action of imaginarily removed part of the outflowing jet [2, pages 47-52]. He expresses it in terms of the pressure $p_{1}$ in the reference section of the imaginarily removed part of the jet: $S=p_{1} \omega_{0}$. Let us assume that $p_{1}=p_{\text {out }}$, where $p_{\text {out }}$ is the hydrostatic pressure of the outer fluid flow. Then 


$$
d S=p_{\text {out }} \frac{d Q}{v}
$$

where $d Q / v$ is the cross-sectional area of the outflowing jet.

Let us draw a schematic diagram of a converging DP (which is tapering) (Fig. 2). For an infinitely short segment $d x$ of the stream in DP with taking into account the force $d S$ (4) which takes the place of the imaginarily removed in the section 3-3 part of the outflowing jet; let us write hydraulic equation of the change in momentum according to the algorithm which is suggested by prof. Yu. M. Konstantinov for collecting pipelines in [7, pages 122-124]:

$$
\begin{aligned}
& \alpha_{0} \rho(Q-d Q)(V-d V)+\alpha_{0} \rho d Q v \cos \varphi-\alpha_{0} \rho Q V=\rho \omega-(p+d p)(\omega-d \omega)- \\
& -\left(p+\frac{d p}{2}\right) d \omega+p_{\text {out }} \frac{d Q}{v} \cos \vartheta-\rho g\left(\omega-\frac{d \omega}{2}\right) \sin \psi \cdot d x-\rho g\left(\omega-\frac{d \omega}{2}\right) d h_{x},
\end{aligned}
$$

Here in Equation (5), projections of the following quantities on the axis $x$ of DP are presented:

$\alpha_{0} \rho Q V$ - the momentum of fluid stream in the reference cross-section 1-1 ( Fig. 2 );

$\alpha_{0} \rho(Q-d Q)(V-d V)$ - ditto in the cross-section 2-2; where $V$ and $Q$ are the average velocity and flow rate of the stream respectively in the cross-section 1-1; $V$ and $Q$ are ditto of the outflowing jet (Fig. 2);

$\alpha_{0} \rho d Q v \cos \varphi$ - the momentum of the out-flowing jet; $\varphi$ is the angle between the average velocity $\vec{V}$ of the main stream in DP and that of the out-flowing jet;

$\rho \omega$ - the momentum of the force of hydraulic pressure in the section 1-1;

$(p+d p)(\omega-d \omega)$ - ditto in the section 2-2; $d p$ is the change of pressure in the interval between the sections $1-1$ and $2-2 ; d \omega$ is the change of free cross-sectional area of the fluid stream in this interval.

$(p+d p / 2) d \omega$ - the longitude component of the momentum caused by the reaction force $\mathrm{R}$ of the walls of the converging pipe in the stream interval between the cross-sections 1-1 and 2-2, $R=\frac{p+(p+d p)}{2} d \omega=\left(p+\frac{d p}{2}\right) d \omega ;$

$p_{\text {out }} \frac{d Q}{v} \cos \vartheta-$ the momentum caused by the force which acts as equivalent of the imaginarily removed part of the jet; $p_{\text {out }}$ is the hydrodynamic pressure of the outer fluid flow in the crosssection $3-3 ; \vartheta$ is the angle between the directions of movement of the main fluid flow inside DP and the force $S$ which acts as equivalent of the imaginarily removed part of the outflow jet (Fig. 2, Fig. 3);

$\rho g\left(\omega-\frac{d \omega}{2}\right) \sin \psi \cdot d x$ - the momentum caused by the own weight of the stream segment of $d x$-length; the minus sign before this term in Equation (5) automatically agrees the direction of momentum projection caused by the force $G$ with the direction of the axis $x$ of the stream 
when the angle $\psi$ changes within $0^{\circ} \ldots 360^{\circ}$;

$\rho g\left(\omega-\frac{d \omega}{2}\right) d h_{x}-$ the momentum of the friction force $T ; \quad T=\tau_{o}\left(\chi+\frac{d \chi}{2}\right) d x=$ $=\rho g\left(R+\frac{d R}{2}\right)\left(\chi+\frac{d \chi}{2}\right) \frac{d h_{x}}{d x} d x=\rho g\left(\omega-\frac{d \omega}{2}\right) d h_{x}$ where $\tau_{o}$ is the mean friction stress at the DP wall, $\tau_{o}=\rho g R \frac{d h_{x}}{d x}[7$, pages 76];

$R$ is the hydraulic radius; $\chi=\omega / R$ is the wetter perimeter of the stream. The angles $\varphi, \vartheta$, and $\psi$ are taken counter-clockwise (see Fig. 2, Fig. 3), their values are within $0^{\circ} \ldots 360^{\circ}$.

From Equation (5), a corrected DEVFRFF is obtained. For cylindrical pipes when $\omega=$ const and $d Q=\omega d V$ it takes the following form:

$$
\frac{\alpha_{0}(v \cos \varphi-2 V) d V}{g}+d \frac{p}{\rho g}+\frac{p_{\text {out }}}{\rho g} \frac{d V}{v} \cos \vartheta+\sin \psi \cdot d x+d h_{x}=0
$$

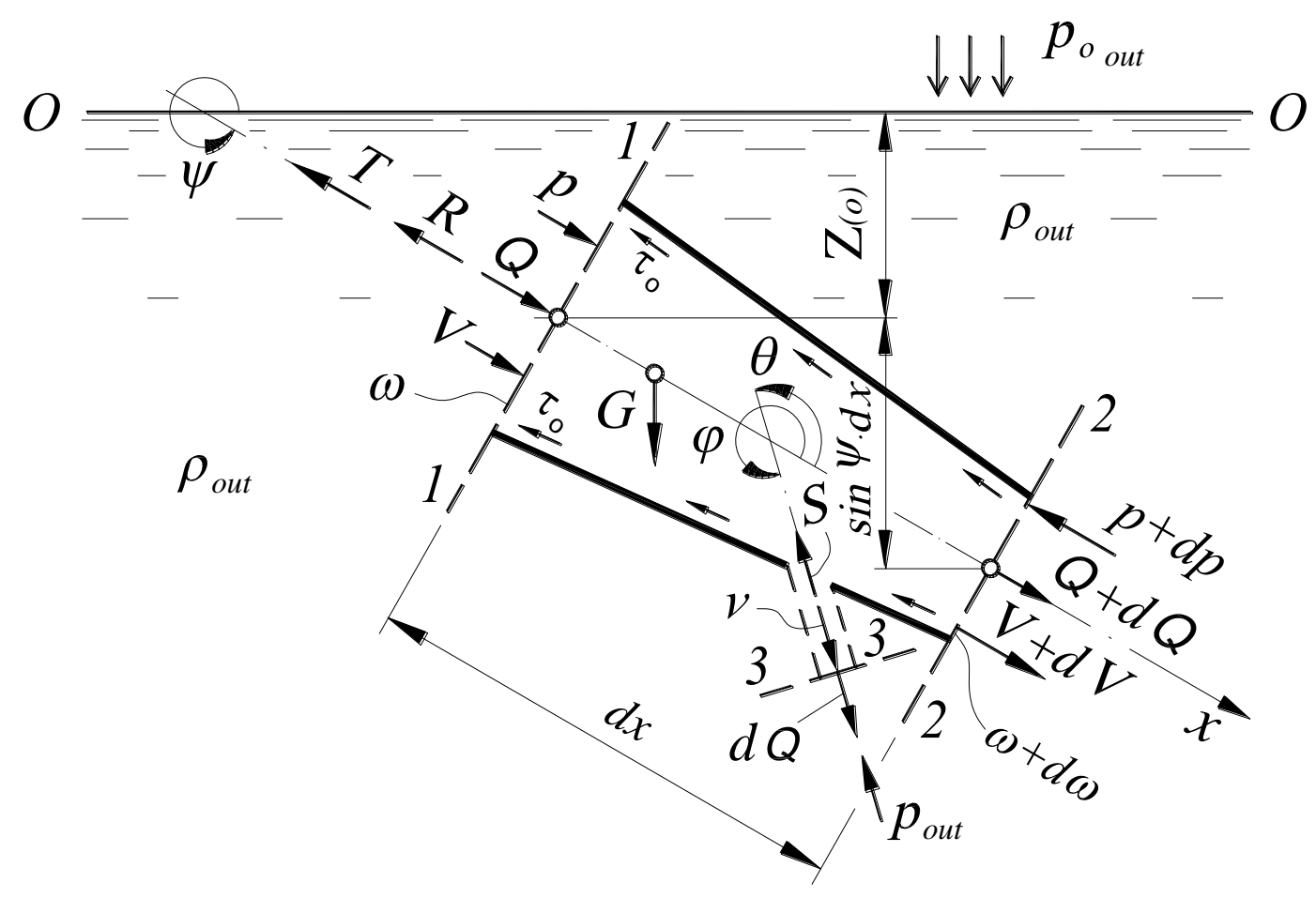

Figure 2: Schematic diagram of enforced stream with mass loss

In Equation (6), the force $S$ is represented by the term $\frac{p_{\text {out }}}{\rho g} \frac{d V}{v} \cos \vartheta$. In previous works of the author which were published before 2009, for example [8], the force $S$ is determined as a 
force of pressure of out-flowing jet upon a flat wall, the velocity $v$ of the jet being perpendicular to the wall: $S=\alpha_{o} \rho v d Q$.

\section{Reduction of DEVFRFF to a single-variable equation}

The main stream inside DP is formed under the influence of separate out-flowing jets. Let us express variables $V, d V, v, d p, p_{\text {out }}, d h_{x}, d x$ in Equation (6) in terms of the full head $H_{(x)}$ under the action of which the jets flow out and, as well as in terms of the independent variable $x$. Let us assume that $m_{(x)}=v_{(x)} \cos \varphi / V_{(x)} \neq$ const $; \quad 0^{\circ} \leq \varphi \leq 360^{\circ} ; \quad 0^{\circ} \leq \vartheta \leq 360^{\circ}$; $0^{\circ} \leq \psi \leq 360^{\circ}$. In different quadrants, the functions $\sin \psi$ and $\cos \varphi, \cos \vartheta, \cos \xi$ are of different signs. In the differential equation, these signs are taken into account automatically.

Thus, the average velocity of a jets flow through a hole in the DP wall

$$
v_{(x)}=\varphi \sqrt{2 g H_{(x)}}=a H_{(x)}^{1 / 2}
$$

where

$$
a=\varphi \sqrt{2 g}=\text { const }, m^{0,5} / s
$$

$\varphi$ is the coefficient of speed; $H_{(x)}$ is the full head under the action of which the jets flow out, it is equal to the difference of the full heads of fluid streams inside and outside the DP:

$$
H_{(x)}=\frac{p_{i n_{(x)}}-p_{\text {out }(x)}}{\rho g} .
$$

The full pressure of internal stream of fluid at the end of the reference dx-long interval of DP:

$$
p_{i n_{(x)}}=-\rho g x \sin \psi+p_{(x)}+\frac{\alpha \rho V_{(x)}^{2}}{2} \cos \varphi .
$$

The full pressure of external fluid stream:

$$
p_{\text {out } x)}=\rho_{\text {out }} g\left(Z_{(0)}-\sin \psi \cdot x\right)+p_{o_{\text {out }}}+\frac{\alpha \rho_{\text {out }} V_{\text {out }}^{2}}{2} \cos \xi
$$

where $\rho$ is the density of fluid inside DP; $\rho_{\text {out }}$ is ditto outside the DP; $Z_{(0)}$ is the depth of submergence of the DP axis at the beginning of the reference interval at $d x=0$ (see Fig. 2); $p_{o_{\text {out }}}$ is the piezometric fluid pressure outside the DP; $\xi$ is the angle between the velocities $\overrightarrow{v_{(x)}}$ of the out-flowing jet (in outer section of outlet) and $\overrightarrow{V_{\text {out }}}$ of the outer stream (Fig. 3); $V_{(x)}$ is the stream velocity inside DP.

For operation of DP, the flowing condition must be satisfied:

$$
p_{i n_{(x)}}>p_{\text {out }(x)}
$$


Let us express the difference between the internal and external fluid pressures in terms of the head of the column of the fluid which the DP is filled of

$$
H_{(x)}=\left(\frac{\rho_{\text {out }}}{\rho}-1\right) \sin \psi \cdot x-\frac{\rho_{\text {out }}}{\rho} Z_{(o)}+\frac{p_{(x)}-p_{o_{\text {out }}}}{\rho g}+\frac{\alpha}{2 g}\left(V_{(x)}^{2} \cos \varphi-\frac{\rho_{\text {out }}}{\rho} V_{\text {out }}^{2} \cos \xi\right) .
$$

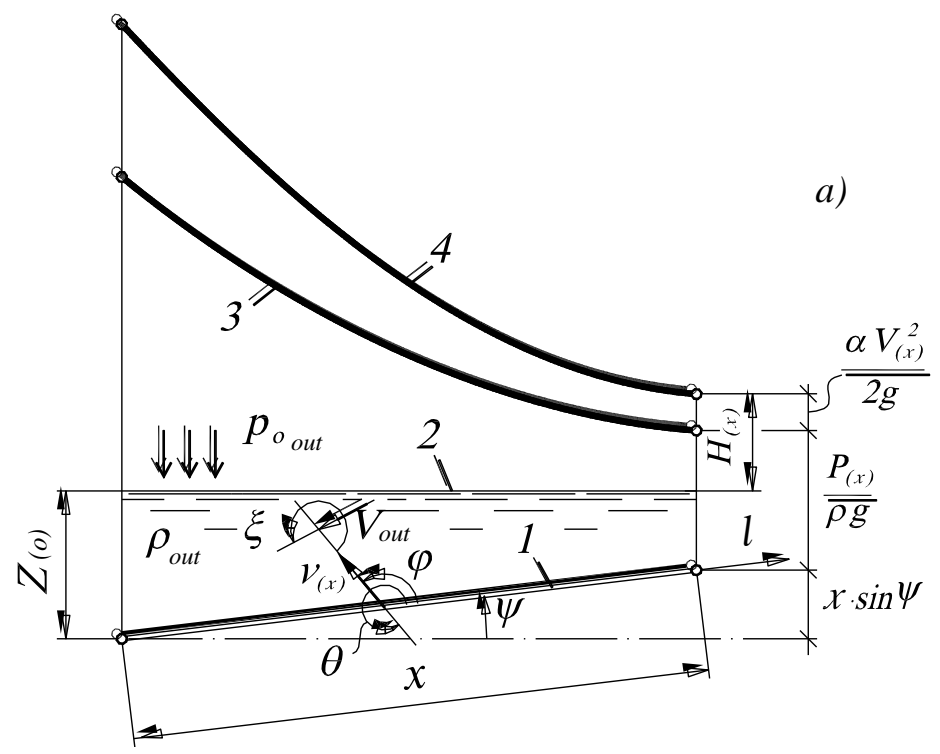

b)

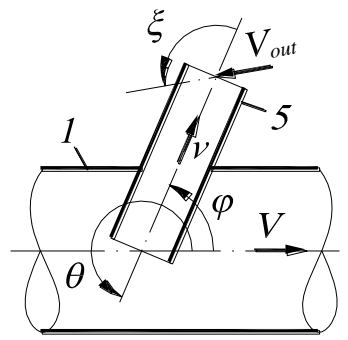

Figure 3: Schematic diagram of DP: (a) heads acting upon DP ; $(b)$ angles between vectors of fluid velocities : 1 - DP; 2 - level of the fluid which washes DP from outside ; 3- piezometric line of stream inside DP; 4 - ditto of full head ; 5 - outlet nozzle; $l$ - axis of DP; $v, V, V_{\text {out }}$ velocities of outflow jet, internal, and external streams respectively.

Let us assume that $V_{\text {out }} \neq f(x) ; p_{o_{\text {out }}} \neq f(x)$. From (13), we find the differential of piezometric head of fluid flow inside DP

$$
d\left(\frac{p_{(x)}}{\rho g}\right)=\left(1-\frac{\rho_{\text {out }}}{\rho}\right) \sin \psi \cdot d x-\frac{\alpha V_{(x)} d V_{(x)}}{g} \cos \varphi+d H_{(x)},
$$

where the multiplier $\cos \varphi$ takes into account the dynamic head of the stream inside DP onto free cross-section of the jet at the intake of the outlet hole. The essence of the multiplier $\cos \varphi$ is clear from Fig. 4.

The differential of the flow rate of the dispensed from DP along the pass

$$
d Q_{d i s t r i b_{(x)}}=n \mu \omega_{h o l e} \sqrt{2 g H_{(x)}} d x=b H_{(x)}^{1 / 2} d x,
$$

where $n$ is the number of outlet holes per unit length of DP, $m^{-1} ; \omega_{h o l e}$ is the area of the hole; $\mu$ is the coefficient of flow rate of this hole 


$$
b=n \mu \omega_{\text {hole }} \sqrt{2 g}=\text { const }, \quad m^{1.5} / \mathrm{s} .
$$

The differential of the flow rate of the main fluid stream inside DP

$$
d Q_{(x)}=-d Q_{d i s t r i b(x)}=-b H_{(x)}^{1 / 2} d x .
$$

The flow rate of dispensed from DP along the path fluid flows inside DP, $Q_{(x)}=\int_{0}^{x} d Q_{(x)}+C=-b \int_{0}^{x} H_{(x)}^{1 / 2} d x+C$. When $x=0, C=Q_{0}$. Thus

$$
Q_{(x)}=Q_{0}-b \int_{0}^{x} H_{(x)}^{1 / 2} d x
$$

where $Q_{(o)}$ is the flow rate at the input of DP, in general case, for a DP of the length L it can be written as $Q_{(0)}=Q_{t r}+b \int_{0}^{L} H_{(x)}^{1 / 2} d x$, where $Q_{t r}$ is the transitional flow rate of fluid at the output of DP.

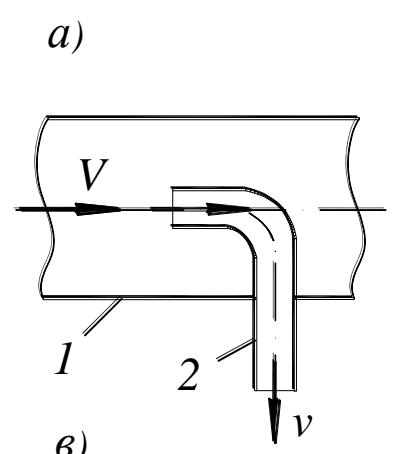

8)

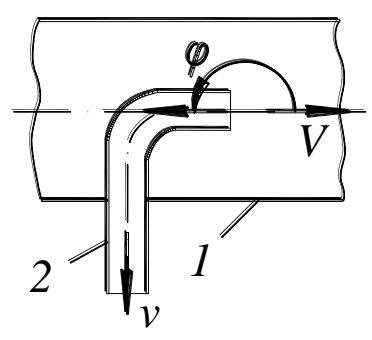

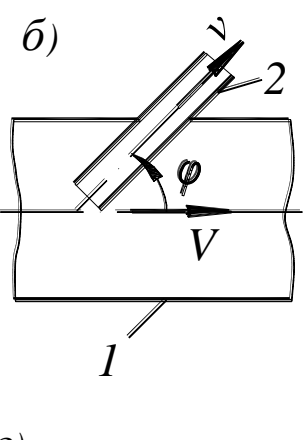

2)

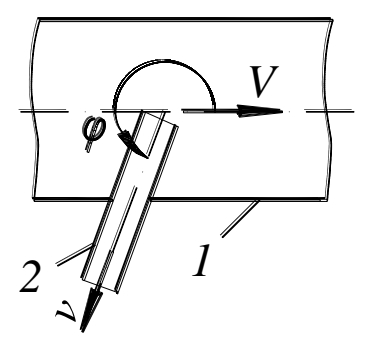

b)

Figure 4: Schematic diagram of action of dynamic head of main stream inside DP upon Surface of free cross-sectional area of stream at input of outlet hole under different values of angle $\varphi: 0^{\circ}-(a) ; \quad 0^{\circ} \ldots 90^{\circ}-(b) ; 180^{\circ}-(c) ; 180^{\circ} \ldots 270^{\circ}-(d)$

The differential of average speed of the fluid stream inside DP is determined from (18)

$$
\text { c) } \left.\quad Q_{(x)}=Q_{0}-b \int_{0}^{x} d\right) \text { ' } x \text {, }
$$


where $\omega$ is the cross-section area of DP stream inside DP.

The average spied of fluid stream inside DP

$$
V_{(x)}=\frac{Q_{(x)}}{\omega}=\frac{1}{\omega}\left(Q_{0}-b \int_{0}^{x} H_{(x)}^{1 / 2} d x\right) .
$$

The differential of the head loss $d h_{x}$ in the infinitely short $d x$ - long interval of cylindrical DP situated between neighboring outlet holes in the DP can be with sufficient exactness determined in the same way as it is for uniform motion i.e. according to Darci-Weisbach formula [7, page 124]

$$
d h_{x}=d\left(\lambda_{(x)} \frac{x}{D} \frac{V_{(x)}^{2}}{2 g}\right)=\frac{1}{2 g \omega^{2} D} d\left[\lambda_{(x)}\left(Q_{o}-b \int_{0}^{x} H_{(x)}^{1 / 2} d x\right)^{2} x\right],
$$

where $D$ is the diameter of DP.

The dependences (7), (11), (14), (19), (20), (21) are to be substituted into Equation (6). The non-linear integro-differential equation of enforced fluid flow with discrete loss in its flow rate along the path for an inclined to horizontal cylindrical DP is obtained by means of this substitution. It is an equation in one unknown function $H_{(x)}$ with taking into account the hydrodynamic pressure of the external fluid flow:

$$
\begin{aligned}
& \frac{1}{2 g \omega^{2} D} d\left[\lambda_{(x)}\left(Q_{0}-b \int_{0}^{x} H_{(x)}^{1 / 2} d x\right)^{2} x\right]-\frac{\alpha_{o} a b \cos \varphi}{g \omega} H_{(x)} d x+d H_{(x)}+ \\
& +\frac{2 \alpha_{o}+\alpha \cos \varphi}{g \omega^{2}}\left(Q_{0}-b \int_{0}^{x} H_{(x)}^{1 / 2} d x\right) b H_{(x)}^{1 / 2} d x+ \\
& +\left(2-\frac{\rho_{\text {out }}}{\rho}\right) \sin \psi \cdot d x-\frac{b \cos \vartheta}{a \omega}\left[\frac{p_{o_{\text {out }}}}{\rho g}+\frac{\rho_{\text {out }}}{\rho}\left(Z_{(0)}-\sin \psi \cdot x+\frac{\alpha V_{\text {out }}^{2}}{2 g} \cos \xi\right)\right] d x=0,
\end{aligned}
$$

where $\lambda_{x)}=f\left(H_{(x)}\right)$, depending on situated in sequence intervals of hydraulic resistance in DP, provided that the flow rate decreases along the main stream. In Equation (22) the hydrodynamic pressure of the external fluid flow is taken into account by means of the

following expressions: $\frac{b \cos \vartheta}{a \omega}\left[\frac{p_{o_{\text {out }}}}{\rho g}+\frac{\rho_{\text {out }}}{\rho}\left(Z_{(0)}-\sin \psi \cdot x+\frac{\alpha V_{\text {out }}^{2}}{2 g} \cos \xi\right)\right] d x$.

By means of the method suggested in the article [8], the equation (22) is solved for laminar flow as well as for all the three intervals of hydraulic resistance of turbulent flow [9]. The obtained calculation dependences have been checked by experiments. Unlike in the known techniques of DP calculating, in the obtained relations, the hydrodynamic pressure of the external fluid flow is taken into account. This improves exactness during designing. 


\section{Conclusion}

In order to improve the exactness of calculation of distributive pipelines, the hydrodynamic pressure of the fluid flow which washes the DP from outside is taken into account in the differential equation of variable flow rate fluid flow for pressure distributive pipelines. The variables of the obtained equation are expressed in terms of full operating head and in terms of independent distance along the axis of the stream. This equation is reduced to a singlevariable equation.

\section{References}

[1] Navoyan, Kh. A. (1975). Examples of calculation for water-passing structures. Kiev.

[2] Petrov, G. A. (1964). Hydraulics of variable mass (variable flow rate fluid flow along the path). Kharkiv: Kh SU.

[3] Danilov, Yu. M. (2004). Investigation of turbulent mixing of two-component mixture in a pipe of periodically variable cross-section. Bulletin of Kazan Technological University. №1, pp. 172179.

[4] Levitsky, B. F., Cherniuk, V. V. (1992). Waste Water Discharge Plant . Author's certificate 1756483 USSR International Classification of Inventions 03F 1/00, 5/12, № 4806326/29, Bulletin № 31.

[5] Cherniuk, V. (1995). Water discharge of periodic operation. In Problemy budownictwa i inżynierii środowiska. Cz. II. Inżynieria Środowiska: praci IV naukowej konferencji, 1995 (pp. 9-14). Rzeszów: Politechnika Rzeszowska.

[6] Egorov, A. I. (1984). Hydraulics of pressure pipe system in water supply purifying structure. Moskow: Stroyizdat.

[7] Konstantinov, Yu. M. (1988). Hydraulics: text-book. Kiev: Vyscha Shkola.

[8] Cherniuk, V. V. (2008). Method of calculation for pressure distributive pipelines. Applied hydromechanics .Volume 10 (82), №3, pp. 65-76. Kiev: Institute of Hydrodynamics of Ukrainian National Academy of Sciences.

[9] Cherniuk, V. V. (2010). Regulation of integral parameters of enforced fluid flows by means of hydrodynamic active additives. Manuscript. Thesis for a scientific degree of doctor of technical sciences by speciality 05.23.16 Hydrodynamics and engineering hydrology, National University of Civil Building and Architecture, Kiev. 